\title{
Energy-momentum dispersion relation of plasmarons in graphene
}

\author{
P. M. Krstajić ${ }^{1}$ and F. M. Peeters ${ }^{2}$ \\ ${ }^{1}$ Institute of Microelectronic Technologies and Single Crystals (IHTM), University of Belgrade, Njegoševa 12, 11000 Belgrade, Serbia \\ ${ }^{2}$ Departement Fysica, Universiteit Antwerpen, Groenenborgerlaan 171, B-2020 Antwerpen, Belgium
}

(Received 27 February 2012; published 31 May 2012)

\begin{abstract}
The many-body correction to the band structure of a quasi-free-standing graphene layer is obtained within the Overhauser approach, where the electron-plasmon interaction is described as a field theoretical problem. We find that the Dirac-like spectrum is shifted by $\Delta E(\mathbf{k}=0)$, which is on the order of $50-150 \mathrm{meV}$, depending on the electron concentration $n_{e}$, and is in semiquantitative agreement with experimental data. The value of the Fermi velocity is renormalized by several percents and decreases with increasing electron concentration as found experimentally.
\end{abstract}

DOI: 10.1103/PhysRevB.85.205454

PACS number(s): 73.22.Pr, 73.20.Mf, 71.10.-w

\section{INTRODUCTION}

There has been growing interest in plasmarons ${ }^{1-4}$ in doped graphene, both from experimental and theoretical research groups. Angle-resolved photoemission spectroscopy (ARPES) has been employed ${ }^{4}$ to probe the band structure of high quality quasi-free-standing and doped graphene. In that study it was found that the well known linear Dirac-like spectrum does not provide a sufficiently detailed picture of the charge carrying excitations. Composite "plasmaron" particles, which are bound states of charge carriers with plasmons, are observed. Furthermore, instead of a single Dirac point three crossing points were detected: ${ }^{4}$ the first between pure charge bands, the second between pure plasmaron bands, and the third one between charge and plasmaron bands. It is well known that in the long wavelength limit the plasmon frequency in graphene is proportional to the square root of the wave vector, ${ }^{5-8}$ and a plasmaronic quasiparticle is formed when the plasmons and charge carriers have the same group velocity. ${ }^{9}$ The reason why this more complicated picture of the band structure has not been observed earlier is due to the low quality and low mobility of old samples of graphene. The spectral function $A(\mathbf{k}, \omega)$ [which is related to the complex selfenergy $\Sigma(\mathbf{k}, \omega)]$ has been used to analyze these experimental data. Since the self-energy $\Sigma(\mathbf{k}, \omega)$ depends on the dielectric function $\epsilon(\mathbf{k}, \omega)$ the plasmons are naturally incorporated in this treatment.

In this paper, we use a very different approach that is based on second order perturbation theory of the electron-plasmon interaction that is treated as a theoretical field problem. Our model is able to determine the correction to the Dirac band structure as due to the interaction of charge carriers with plasmons. For the interaction between plasmons and charge carriers we generalize the Overhauser approach ${ }^{9,10}$ to the two-dimensional electron gas (2DEG) in graphene. The fact that the plasmon excitation $\omega_{\mathbf{q}}$ of the Dirac sea remains well defined even when it enters the interband particle-hole continuum $^{3}$ gives credibility to the method used. This is especially important when the momentum $q$ in the integration is large. The value of the shift at $\mathbf{k}=0, \Delta E(0)$, is in semiquantitative agreement with recent experimental data. ${ }^{4}$

We organize the paper as follows. In Sec. II we present the theoretical model and derive relevant expressions for the interaction and the coupling between electrons and plasmons in graphene. In the subsequent section, Sec. III, the numerical calculations of the energy correction due to the interaction with plasmons are presented for various doping levels, i.e., charge carrier density. The influence of the electron concentration is analyzed and discussed. Finally, we summarize our results and present the conclusions in Sec. IV, where also a comparison is made with recent experiments.

\section{THEORETICAL MODEL}

The massless (noninteracting) Dirac band Hamiltonian of graphene is given by

$$
H=\hbar v_{F} \boldsymbol{\sigma} \cdot \mathbf{k},
$$

where $v_{F}$ is the Fermi velocity, and $\sigma=\left(\sigma_{x}, \sigma_{y}\right)$ are the two-dimensional (2D) Pauli matrices. Plasmons in a single layer graphene have been investigated theoretically. ${ }^{5-7}$ Their dispersion are determined by zeros of the dielectric function, and in the long wavelength limit it is given by $\omega_{\mathbf{q}}=\sqrt{\alpha}\left(g_{s} g_{v} \pi n_{s}\right)^{1 / 4} v_{F} \sqrt{q}$, where $g_{s}=2$ and $g_{v}=2$ are the degeneracy factors for spin and valley degrees of freedom, respectively. The excitations will be described by a scalar field previously described by Overhauser ${ }^{9}$ for the three-dimensional (3D) electron gas. The changes in the electron spectrum are calculated analogously as for a polaron problem where a test electron interacts with the plasmon field. The interaction of a remote electron (i.e., an electron displaced from the graphene layer) with plasmons was treated in our earlier work, ${ }^{10}$ and the interaction term of the Hamiltonian is given by

$$
H_{\text {int }}=\sum_{\mathbf{q}} \frac{V_{\mathbf{q}}}{\sqrt{\Omega}} \exp (i \mathbf{q} \cdot \mathbf{r})\left(a_{\mathbf{q}}+a_{-\mathbf{q}}^{\dagger}\right),
$$

where the electron-plasmon (interaction) matrix element $V_{\mathbf{q}}$ is ${ }^{10}$

$$
V_{\mathbf{q}}=\frac{2 \pi e^{2}}{\kappa} \sqrt{\frac{n_{s}^{\prime} g_{v}}{\hbar \omega_{\mathbf{q}}^{\prime}} \frac{W}{8 \pi}} .
$$

Here $n_{s}^{\prime}$ is the concentration per unit cell $n_{s}^{\prime}=n_{e} 3 \sqrt{3} / 2 a_{\mathrm{C}-\mathrm{C}}^{2}$, where $a_{\mathrm{C}-\mathrm{C}}=1.42 \AA$ is the carbon-carbon distance. Further, $W$ is the bandwidth of the conduction band, and determines the momentum cutoff. Within the tight-binding picture the value of $W$ is $\sqrt{\sqrt{3} \pi} t$, where $t$ is the hopping energy ${ }^{11} t=2.8 \mathrm{eV}$. The same value of the energy cutoff was used by LeBlanc et al. ${ }^{1}$ 
while calculating the density of states. We emphasize at this point that $\omega_{\mathbf{q}}^{\prime}$ is not the bare plasmon frequency but is modified by the polarization of the electron gas and is given by ${ }^{10}$

$$
\omega_{\mathbf{q}}^{\prime 2}=\omega_{\mathbf{q}}^{2} \frac{\epsilon(q)}{\epsilon(q)-1} .
$$

It can be shown that in the long wavelength limit $(q \rightarrow 0)$ and within the random phase approximation (RPA) the (static) dielectric function can be approximated by ${ }^{6}$

$$
\epsilon(q)=1+\frac{q_{s}}{q},
$$

where $q_{s}$ is the screening wave vector, $q_{s}=g_{s} g_{v} e^{2} k_{F} /\left(\kappa \hbar v_{F}\right)$. The previous relation is obtained from the general relation $\epsilon(q, \omega)=1+v_{c}(q) \Pi(q, \omega)$, where $v_{c}(q)=2 \pi e^{2} /(\kappa q)$ is the Fourier transform of the 2D Coulomb interaction, and $\Pi(q, \omega)$ is the $2 \mathrm{D}$ polarizability.

Now, one may readily evaluate the correction in the energy band due to the interaction between the electrons and the plasmons. This will be done by employing second order perturbation theory, and for the case of graphene it reads

$$
\Delta E_{0}(\mathbf{k})=-P \frac{1}{\Omega} \sum_{\mathbf{q}} \frac{\left|V_{\mathbf{q}}\right|^{2}}{\hbar \omega_{\mathbf{q}}+\hbar v_{F}|\mathbf{k}-\mathbf{q}|-\hbar v_{F}|\mathbf{k}|},
$$

where $P(\cdot)$ stands for the principal value. However, this correction is done within nondegenerate Rayleigh-Schrödinger perturbation theory (RSPT). Because of degeneracy, the improved Wigner-Brillouin perturbation theory (IWBPT) needs to be used. ${ }^{12}$ The main idea is to ensure improved convergence when the denominator in Eq. (6) is close to zero, which is realized by adding the term $\Delta=\Delta E(\mathbf{k})-\Delta E_{0}(\mathbf{k})\left[\Delta E_{0}(\mathbf{k})\right.$ is evaluated within RSPT],

$$
\Delta E(\mathbf{k})=-P \sum_{\mathbf{q}} \frac{\left|V_{\mathbf{q}}\right|^{2}}{\hbar \omega_{\mathbf{q}}+\hbar v_{F}|\mathbf{k}-\mathbf{q}|-\hbar v_{F}|\mathbf{k}|-\Delta(\mathbf{k})} .
$$

It is obvious that Eq. (7) should be solved self-consistently since $\Delta E$ appears on both sides of the equation. In the next section the relation for $\Delta E(\mathbf{k})$ will be calculated for concrete values of the doping level, permittivity, and other parameters of the material.

At this point, we address the influence of the broadening of plasmon excitation, $\Gamma(q)$ when it enters the interband particle-hole continuum. It is defined as $\Gamma(q)=$ $\Gamma\left(q, \omega=\omega_{p l \mathbf{q}}\right)$, where $^{13}$

$$
\Gamma(q, \omega)=\frac{\operatorname{Im}[\Pi(q, \omega)]}{\partial \operatorname{Re}[\Pi(q, \omega)] / \partial \omega} .
$$

For graphene the polarizability function can be approximated as $D_{0}\left(\hbar v_{F} q\right)^{2} /\left(2 \omega^{2}\right)\left[1-\omega^{2} /\left(4 E_{F}^{2}\right)\right]$ for $\omega>v_{F} q$, where $D_{0}$ is the density of states. This form suggests that the imaginary part is zero, so that the broadening can be neglected. This contrasts the case of a 2 DEG with a parabolic energy spectrum, where the polarizability function, given as $\Pi(q, \omega)=$ $q^{2} /\left(4 \sqrt{v_{F}^{2} q^{2}-\omega^{2}}\right)$, can easily assume a large imaginary $\operatorname{part}^{11}$ when $\omega>v_{F} q$. As pointed out in Ref. 3, the plasmon excitation of the Dirac sea remains remarkably well defined even when it enters the interband particle-hole continuum.
This is due to the fact that transitions near the bottom of the interband particle-hole continuum have nearly parallel $\mathbf{k}$ and $\mathbf{k}+\mathbf{q}$ and therefore little charge-fluctuation weight is attached to it. In reality, the damping can be important for very large momentum $q$, but then the contribution to the energy shift, i.e., to the integral in Eq. (7), is small.

\section{NUMERICAL RESULTS}

The numerical calculations are carried out for a doped graphene layer, with electron concentration in the range $n \in$ $(1,10) \times 10^{13} \mathrm{~cm}^{-2}$, corresponding to the Fermi level $E_{F} \in$ $(0.37,1.2) \mathrm{eV}$. While evaluating Eq. (7), it is important to bear in mind that the momentum $q$ has a cutoff value determined by the bandwidth $W$. This was also done when evaluating ${ }^{10}$ the interaction strength $V_{\mathbf{q}}$. Unlike the case of polarons in conventional semiconductors, here it is not straightforward to derive any approximate relation for small $\mathbf{k}$, since plasmons have a complicated dispersion relation, and the interaction matrix element $V_{\mathbf{q}}$ depends on $q$ in a nontrivial manner. Thus we will treat Eq. (6) numerically. Due to conical band structure $\Delta E(\mathbf{k})=\Delta E(k)$, and one may write for small $k$

$$
\Delta E(k)=\Delta E(0)+\alpha k+\beta k^{2} .
$$

In Fig. 1 we show the result for the energy correction $\Delta E(k)$ versus the momentum $k$ for two electron concentrations $n_{e}=10^{13} \mathrm{~cm}^{-2}$ and $n_{e}=3 \times 10^{13} \mathrm{~cm}^{-2}$ (solid curves). On the same graph, the energy correction $\Delta E_{0}(k)$ calculated within RSPT is also given by dashed and dashed-dotted curves. The absolute value of $\Delta E(k)$ increases with the electron concentration. Note that the value of $\Delta E$ is not directly related to $\delta E=E_{2}-E_{0}$ as considered in Refs. 1,2 , and 4 , but it is on the order of $\delta E / 2$. The energies $E_{2}$ and $E_{0}$ correspond to the Dirac crossing of the bands of opposite chirality. The shift $\Delta E$ is -23 and $-60 \mathrm{meV}$ for electron concentrations $n_{e}=$ $10^{13} \mathrm{~cm}^{-2}$ and $n_{e}=3 \times 10^{13} \mathrm{~cm}^{-2}$, respectively. Further, the values of the parameters $\alpha$ and $\beta$ are $\alpha=-0.005\left(\hbar v_{F}\right)$, $\beta=-7.4 \times 10^{3}\left(\hbar v_{F}\right)^{2}\left(\mathrm{eV}^{-1}\right)$ for $n_{e}=10^{13} \mathrm{~cm}^{-2}$ and $\alpha=$ $-0.013\left(\hbar v_{F}\right)$, and $\beta=-7.6 \times 10^{3}\left(\hbar v_{F}\right)^{2}\left(\mathrm{eV}^{-1}\right)$ for $n_{e}=$ $3 \times 10^{13} \mathrm{~cm}^{-2}$. These parameters are obtained by fitting Eq. (9) to our numerical values (see Fig. 1) in the range $0<k<0.6 \mathrm{~nm}^{-1}$.

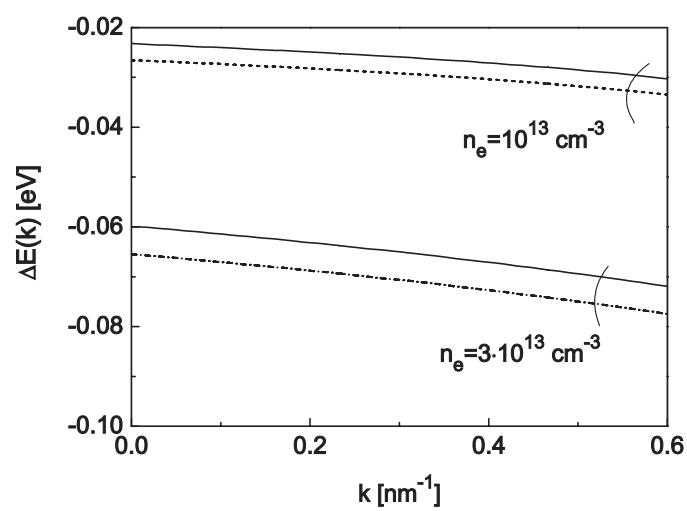

FIG. 1. The correction to the energy $\Delta E(k)$ vs electron momentum $k$ for two values of the doping level $n_{e}=10^{13} \mathrm{~cm}^{-2}$ and $3 \times 10^{13} \mathrm{~cm}^{-2}$ (solid curves). Dashed and dashed-dotted curves correspond to the correction $\Delta E_{0}(k)$ within RSPT. 


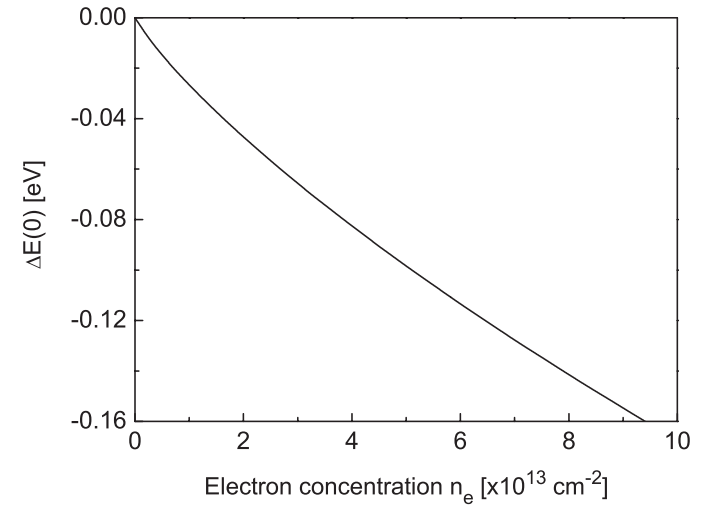

FIG. 2. The correction to the energy $\Delta E(0)$ for $k=0$ vs the electron concentration $n_{e}$.

In Fig. 2 we present the result for the energy correction $\Delta E(0)$ at $k=0$ versus doping of graphene, i.e., the electron concentration $n_{e}$. As can be seen, the absolute value of $\Delta E(0)$ increases with the electron concentration. This is mainly the consequence of the dependence of the matrix element $V_{\mathbf{q}}$ on $n_{e}$ [see Eq. (3)]. The obtained dependence of the energy shift on the concentration could be fitted to $\Delta E(0)=c_{1} n_{e}+c_{2} n_{e}^{2}$, where $c_{1}=-2.14 \times 10^{-15} \mathrm{~cm}^{2} \mathrm{eV}$ and $c_{2}=5.39 \times 10^{-30} \mathrm{~cm}^{4} \mathrm{eV}$.

In Fig. 3 we show how the fitting parameter $\alpha$ in Eq. (9) changes with the electron concentration $n_{e}$. The absolute value of $\alpha$ increases with the concentration, since the matrix element $V_{\mathbf{q}}$ depends on $n_{e}$ as $\sqrt{n_{e}}$ [see Eq. (3)]. The negative value of $\alpha$ implies a lower effective value of the Fermi velocity, and thus the electron velocity decreases with doping. Our numerical results could be fitted to the expression $\alpha /\left(\hbar v_{F}\right)=d_{1} n_{e}+d_{2} n_{e}^{2}$, where $d_{1}=-3.8 \times 10^{-16} \mathrm{~cm}^{-2}$ and $d_{2}=1.2 \times 10^{-30} \mathrm{~cm}^{-4}$. The quadratic term in $\Delta E(k)$ is shown in Fig. 4 as function of the electron concentration.

\section{CONCLUSION AND COMPARISON WITH EXPERIMENT}

In this paper we analyzed the interaction between an electron and the collective excitation of the electron gas, i.e., plasmons in graphene, by using a field-theoretical approach. This interaction is modeled by generalizing the Overhauser approach $^{9}$ to the 2DEG in graphene. We evaluated the

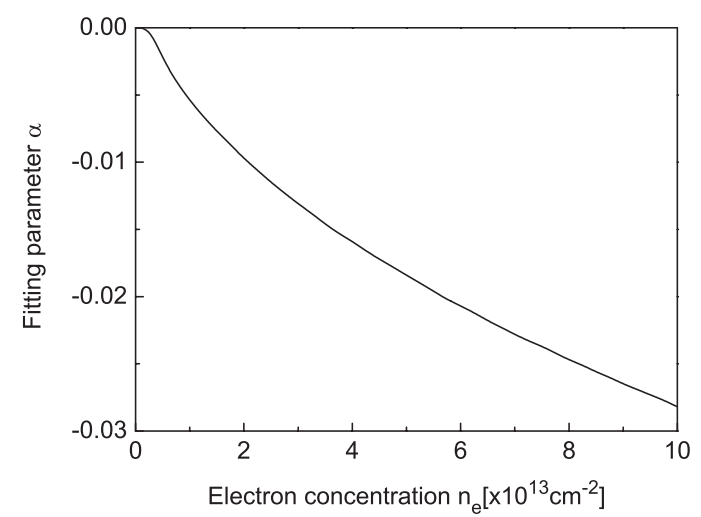

FIG. 3. The fitting parameter $\alpha$ vs the electron concentration $n_{e}$.

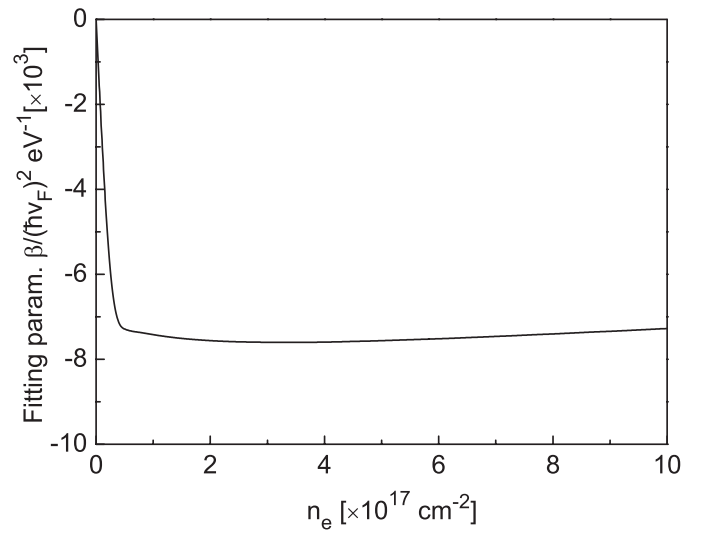

FIG. 4. The fitting parameter $\beta$ vs the electron concentration $n_{e}$.

energy correction as a result of this interaction. Second order perturbation theory was employed to determine the energy of the plasmaron, which is a composite quasiparticle, i.e., a bound state of an electron with plasmons.

The motivations for this study are very recent experimental findings ${ }^{2,4}$ that show that the existence of plasmarons and its band cannot be neglected, at least not in clean samples of quasi-free-standing doped graphene. These experimental studies showed that neither defect scattering nor the symmetry breaking potential induced by the substrate are responsible for the deviations from the accepted conical band structure with one crossing point at $E_{D}=0$. The numerical results for $\Delta E(0)$ obtained in this paper are of the same order as the corresponding experimental value ${ }^{4}$ for $n$-doped graphene, where the bands are shifted by a value of about $0.25 \mathrm{eV}$ for electron density $n_{e}=1.7 \times 10^{13} \mathrm{~cm}^{-2}$. Better agreement is achieved when we take a larger value for the cutoff energy $W$. Note, however, that the exact value of the energy cutoff is not known, but is usually estimated from the tight-binding model. Furthermore, it was shown that for small and finite $k, \Delta E(k)$ can be approximated by a linear function $\alpha k$, which in turn renormalizes the value of the Fermi velocity $v_{F} \rightarrow(1+\alpha) v_{F}$ $\left[\alpha=-1 \%\right.$ to $3 \%$ for $\left.n_{e} \in(1,10) \times 10^{13} \mathrm{~cm}^{-2}\right]$ for the plasmaronic band. There are already a few theoretical works ${ }^{14,15}$ devoted to many-body corrections to the Fermi velocity, and its dependence on the doping level. In Ref. 15 it was estimated that $v_{F}$ may decrease up to $8 \%$ for $n_{e}=4 \times 10^{13} \mathrm{~cm}^{-2}$. In addition, there are indirect experimental data that suggest a small but noticeable modulation of the Fermi velocity with doping. ${ }^{16}$ Quite recently, the change of the velocity with electron concentration is observed in suspended graphene, ${ }^{17}$ through cyclotron resonance measurements. Here, we found that the absolute value of $\alpha$ increases with the bandwidth $W$, while the coefficient $\beta$ decreases with $W$, which means that the energy band dispersion is more linear for larger values of the momentum cutoff. On the other hand, the value of $\Delta E(0)$ is in even better agreement with $a b$ initio studies of graphene. ${ }^{18}$ The authors of Ref. 18 performed a first-principles calculation in order to simulate the angle-resolved photoemission spectra of graphene. They reported values of $\Delta E(0)$ for two electron concentrations $n_{e}=4.5 \times 10^{13}$ and $n_{e}=1.2 \times 10^{14}$ (Fig. 3 in Ref. 18) and for two cases: suspended graphene and with a model substrate. The values for suspended graphene 
are consistent with our theoretical estimates of -85 and $-180 \mathrm{meV}$. The discrepancy with the experimental data for $\Delta E(0)$ could be due to the influence of the substrate, which may even produce a small band gap. However, the agreement for the Fermi velocity with Ref. 18 is not as good. For instance, for $n_{e}=1.2 \times 10^{14}$, the velocity $v_{F}$ reported, is below the value for undoped graphene by more than $10 \%$. This is larger than our estimate of $|\alpha|=3 \%$. The discrepancy is probably due to the fact that in Ref. 18 electron-electron interaction is also taken into account, which is important for high electron concentration.

Finally, we investigated the influence of the doping level on the shift $\Delta E(0)$, and it is shown that it increases with $n_{e}$, in agreement with experiments. Further, it was determined that the shift decreases with an increase of the substrate effective dielectric constant $\epsilon_{S}$, since $V_{\mathbf{q}} \propto 1 / \epsilon\left[\epsilon=\left(1+\epsilon_{S}\right) / 2\right]$, in line with Ref. 2 . Further, the value of $\Delta E(0)$ agrees well with $a b$ initio studies of suspended graphene ${ }^{18}$ for the two electron concentrations. In addition to the existing theoretical studies of the spectral function $A(k, \omega)$, the present paper presents an alternative approach to explain the physical nature of the energy shift and on the more complicated energy band structure in graphene. We realized this by considering the electron-plasmon interaction as described by the matrix element $V_{\mathbf{q}}$, Eq. (3), where the dependence on doping, permittivity and other parameters are explicitly included.

\section{ACKNOWLEDGMENT}

This work was supported by the Flemish Science Foundation (FWO-Vl), the ESF-EuroGRAPHENE project CONGRAN, and the Serbian Ministry of Education and Science (project No. TR 32008).
${ }^{1}$ J. P. F. LeBlanc, J. P. Carbotte, and E. J. Nicol, Phys. Rev. B 84, 165448 (2011).

${ }^{2}$ A. L. Walter, A. Bostwick, K.-J. Jeon, F. Speck, M. Ostler, T. Seyller, L. Moreschini, Y. J. Chang, M. Polini, R. Asgari, A. H. MacDonald, K. Horn, and E. Rotenberg, Phys. Rev. B 84, 085410 (2011).

${ }^{3}$ M. Polini, R. Asgari, G. Borghi, Y. Barlas, T. Pereg-Barnea, and A. H. MacDonald, Phys. Rev. B 77, 081411 (2008).

${ }^{4}$ A. Bostwick, F. Speck, T. Seylerr, K. Horn, M. Pollini, R. Asgai, A. H. MacDoanld, and E. Rotenberg, Science 328, 999 (2010).

${ }^{5}$ E. H. Hwang and S. Das Sarma, Phys. Rev. B 80, 205405 (2009).

${ }^{6}$ E. H. Hwang and S. Das Sarma, Phys. Rev. B 75, 205418 (2007).

${ }^{7}$ S. Das Sarma and E. H. Hwang, Phys. Rev. Lett. 102, 206412 (2009).

${ }^{8}$ M. Jablan, M. Soljačić, and H. Buljan, Phys. Rev. B 83, 161409(R) (2011).

${ }^{9}$ A. W. Overhauser, Phys. Rev. B 3, 1888 (1971).
${ }^{10}$ P. M. Krstajić and F. M. Peeters, Phys. Rev. B 85, 085436 (2012).

${ }^{11}$ A. H. C. Neto, F. Guinea, N. M. R. Peres, K. S. Novoselov, and A. K. Geim, Rev. Mod. Phys. 81, 109 (2009).

${ }^{12}$ F. M. Peeters, P. Warmenbol, and J. T. Devreese, Europhys. Lett. 3, 1219 (1987).

${ }^{13}$ S. M. Badalyan and F. M. Peeters, Phys. Rev. B (2012).

${ }^{14}$ C. Attaccalite and A. Rubio, Phys. Status Solidi B 246, 2523 (2009).

${ }^{15}$ C.-H. Park, F. Giustino, M. L. Cohen, and S. G. Louie, Phys. Rev. Lett. 99, 086804 (2007).

${ }^{16}$ C.-H. Park, F. Giustino, J. L. McChesney, A. Bostwick, T. Ohta, E. Rotenberg, M. L. Cohen, and S. G. Louie, Phys. Rev. B 77, 113410 (2008); J. L. McChesney, A. Bostwick, T. Ohta, T. Seyller, K. Horn, J. Gonzalez, and E. Rotenberg, Phys. Rev. Lett. 104, 136803 (2010).

${ }^{17}$ D. C. Elias, R. V. Gorbachev, A. S. Mayorov, S. V. Morozov, A. A. Zhukov, P. Blake, L. A. Ponomarenko, I. V. Grigorieva, K. S. Novoselov, F. Guinea, and A. K. Geim, Nat. Phys. 7, 701 (2011).

${ }^{18}$ C.-H. Park, F. Giustino, C. D. Spataru, M. L. Cohen, and S. G. Louie, Nano Lett. 9, 4234 (2009). 